\title{
Algunas valoraciones del profesorado respecto a su función tutorial
}

\section{Teaching staff's perceptions regarding their tutorial function}

\author{
María González Álvarez ${ }^{1}$ \\ maria.gz.az@gmail.com, https://orcid.org/0000-0001-6830-579X \\ IES "La Quintana" (Ciaño-Langreo) Asturias, España
}

\section{Resumen:}

La función tutorial se considera en la literatura como un componente básico de la práctica docente y factor de calidad de la enseñanza. Nuestro objetivo en este trabajo es analizar distintos aspectos de la tutoría de los centros de Educación Infantil, Educación Primaria, Educación Secundaria Obligatoria, Bachillerato y FP. Para ello hemos elaborado una prueba que ha sido enviada por correo electrónico a una muestra de tutores y tutoras de todas las comunidades de España y que ha sido cumplimentada por 1350 de ellos. Ante los constantes cambios de la realidad socioeducativa hemos realizado un trabajo exploratorio para obtener información sobre la actual situación de la función tutorial. El análisis de sus respuestas nos indican que, tal como aparece en distintas investigaciones, se sienten satisfechos en su tarea a la vez que consideran que la misma es bastante importante para la educación del alumnado y el funcionamiento del centro. Se sienten razonablemente reconocidos en su función tutorial por parte del profesorado del centro y de las familias, no opinando lo mismo respecto al reconocimiento que les otorgan las administraciones educativas. Entienden que

\begin{abstract}
:
In existing literature the tutorial function is considered as a basic component of the teaching practice and a factor of teaching quality. The goal of our work is to analyze the different aspects of mentoring in Infant, Primary and Secondary Education, Baccalaureate and Vocational Training. In order to achieve this goal, we emailed a test to a sample of mentors in all the autonomous regions of Spain. The test was filled in by 1,350 mentors. Given the constant changes in Spain's socio educational reality, an exploratory work was carried out to obtain information about the current situation of the mentoring function. The analysis of their answers indicates that, as stated in existing research, they feel satisfied with their task, while they consider the aforementioned function is quite important for the education of pupils and the well-functioning of educational institutions. They feel reasonably recognized in their mentoring function both by teachers and by families. Nevertheless, they do not think the same about the recognition shown by the educational authorities. They understand that their mentoring task should count more towards their professional career. They consider that they receive
\end{abstract}

1 Dirección para correspondencia (correspondence address):

María González Álvarez. Profesora de Educación Secundaria. IES “La Quintana" (Ciaño-Langreo) Asturias. C/ Cean Bermúdez n² 2․ 33208 Gijón (España). 
Algunas valoraciones del profesorado respecto a su función tutorial

María González Álvarez

su tarea tutorial debe ser tenida en cuenta en su carrera profesional. Consideran que reciben una adecuada colaboración en sus funciones por parte del profesorado del equipo docente, familias y equipo directivo del centro. Entendemos que las administraciones educativas deberían prestar más apoyo a tutores y tutoras y mejorar su situación profesional y que procede analizar el desarrollo de la acción tutorial en función de los constantes cambios socioeducativos y normativos que se producen.

\section{Palabras clave:}

Función tutorial; satisfacción laboral; funcionamiento del centro; reconocimiento de la acción tutorial; colaboración con tutores. adequate collaboration from the teaching staff, families and management boards in education centres. We understand that the educational authorities should further support mentors and improve their professional situation. Finally, it seems necessary to analyze the development of tutorial action according to the constant socioeducational and normative changes that occur.

\section{Key words:}

Tutor's tasks; work satisfaction; running of the school; tutor recognition; cooperation with tutors.

\section{Résumé:}

La fonction de tutorat est considérée dans la littérature comme un élément fondamental de la pratique de l'enseignement et du facteur de qualité de l'enseignement. Notre objectif dans ce travail est d'analyser différents aspects du tutorat des centres d'éducation infantile, d'enseignement primaire, d'enseignement secondaire obligatoire, de baccalauréat et de formation professionnelle. Pour cela nous avons développé un test qui a été envoyé par email à un échantillon de tuteurs et tutrices de toutes les communautés en Espagne et qui a été complété par 1350 d'entre eux. Compte tenu des changements constants dans la réalité socio-éducative, nous avons fait un travail exploratoire pour obtenir des informations sur la situation actuelle de la fonction tutorielle. L'analyse de leurs réponses indique que, comme cela apparaît dans différentes enquêtes, ils se sentent satisfaits dans leur tâche tout en considérant que c'est très important pour l'éducation des étudiants et le fonctionnement du centre. Ils se sentent raisonnablement reconnus dans leur fonction de tutorat par les enseignants du centre et les familles, ne donnant pas le même avis sur la reconnaissance accordée par les administrations éducatives. Ils comprennent que leur tâche de tutorat devrait être prise en compte dans leur carrière professionnelle. Ils considèrent qu'ils bénéficient d'une collaboration adéquate dans leurs fonctions par le personnel enseignant de l'équipe pédagogique, les familles et l'équipe de gestion du centre. Nous comprenons que les administrations éducatives devraient fournir plus de soutien aux tuteurs et aux tutrices et améliorer leur situation professionnelle et qu'il est nécessaire d'analyser le développement de l'action didactique basée sur les changements socioéducatifs et normatifs constants qui ont lieu.

\section{Mots-clés:}

Fonction tutorielle; satisfaction au travail; fonctionnement du centre; reconnaissance de I'action tutorale; collaboration avec les tuteurs.

Fecha de recepción: 15-10-2017

Fecha de aceptación: 11-10-2018 


\section{Introducción}

En el informe 15 Claves de Análisis para Apuntalar la Agenda Educativa 2030. OIE-UNESCO (Opertti, 2017) se señala que los sistemas educativos se organizan a partir de la centralidad del docente como efectivo orientador y del alumno como genuino protagonista de los aprendizajes. Para Barber y Mourshed (2007) el principal impulsor de las variaciones en el aprendizaje escolar es la calidad de los docentes. Docencia que, comprometida con el proceso de aprendizaje del alumnado, debe tener en cuenta a la tutoría como parte importante de su acción (García-Nieto, 2011).

La tutoría es un componente básico de la práctica docente y factor de calidad de la enseñanza (Álvarez, 2017; Cañas, Campy y Pantoja, 2005; López, 2013). Para Álvarez, "la realidad socio-educativa se encuentra en constantes cambios (...) lo cual requiere, asimismo, un cambio en los roles y funciones de la orientación y la tutoría" (2017: 28-29). Por estos motivos entendemos que, después de haberse regulado por las administraciones educativas la función tutorial y la orientación, procede analizar su situación actual en las enseñanzas no universitarias en España. Para ello hemos realizado un estudio exploratorio de determinados aspectos de dicha función, solicitando a tutores y tutoras sus opiniones y valoraciones respecto a algunas de sus tareas ejercidas en centros de Educación Infantil, Educación Primaria, ESO, Bachillerato y FP del conjunto de las comunidades autónomas de España.

En este estudio tenemos en cuenta diversas variables tales como el género, la edad, el tiempo de experiencia docente, tiempo de experiencia como tutor o tutora, titularidad del centro, comunidad autónoma, número de alumnos y alumnas del centro y horas semanales dedicadas a la tutoría. Aspectos fundamentales a analizar en este trabajo son los referidos a la satisfacción que señalan tener en su actividad tutorial y la importancia que según su opinión tiene la tutoría en la educación del alumnado y en el funcionamiento del centro. Analizamos el reconocimiento que reciben por parte de las administraciones educativas, profesorado y familias del centro sobre su trabajo en la tutoría. Atendemos a la información que nos otorgan tutores y tutoras respecto a que se tenga en cuenta en su carrera profesional el ejercicio de dicha función, así como la retribución económica que pudiera corresponderles. Es importante conocer las valoraciones que realizan respecto a la colaboración 
que tienen en su trabajo por parte del equipo docente, familias y equipo directivo del centro.

\section{Marco teórico}

Entiende Del Valle (2004) que a partir de la Ley Orgánica 1/1990, en España, la tutoría y orientación del alumnado forman parte de la función docente. Función tutorial que debe ser un proceso continuo y sistemático de la acción educativa y que facilite el proceso de aprendizaje y alcance el desarrollo personal del alumnado (Álvarez, 2017; Ceballos, 2017)

La acción tutorial para diversos autores (Álvarez 2017; Mateo 2008 y Rodríguez y Romero 2015), debe ser una acción colectiva y coordinada de todo el profesorado, por lo cual debe realizarse un nuevo enfoque más comprensivo e integral de la tutoría. Para Valenzuela y Sales (2016) los tutores coordinan el trabajo del equipo de profesores y profesoras que atiende al mismo grupo de alumnos y alumnas favoreciendo una práctica educativa coherente y un enfoque globalizador de la acción educativa. Señalan, asimismo, González-Palacios y Avelino-Rubio (2016) que debe dejarse de hablar en singular de tutoría para referirnos a tutorías.

Según Álvarez-Justel (2015) la tutoría no ha estado totalmente integrada en la formación del estudiante, careciendo en muchas ocasiones de objetivos y contenidos concretos, siendo insuficiente la disponibilidad del horario del profesorado y del alumnado para la misma y que en demasiadas ocasiones se reduce a un servicio burocrático.

En aspectos concretos de la enseñanza como es la transición del alumnado de las escuela del medio rural a la educación secundaria se destaca la importancia de la acción tutorial, tanto en el comienzo del nuevo curso como al finalizar la etapa de educación primaria (Bereziartua, Intxausti y Odriozola, 2017). En su trabajo sobre la tutoría y la relación profesor-alumno en la formación para la inserción laboral García (2006) destaca la importancia del apoyo tutorial para favorecer la adquisición de competencias personales, sociales, académicas y laborales. Otro tema de interés que analizan Santana y Feliciano (2006) es el relativo a la actitud y competencia del tutor o tutora, según su criterio la tutoría no es una tarea mecánica ni cualquier profesor o profesora pueden Ilevarla a cabo. Para Bolívar (2007) debe propiciarse una auténtica 
profesionalización del docente que debe conducir a la de un profesional del aprendizaje, al tiempo que un educador.

Respecto al futuro de la acción tutorial Quintanal (2006) entiende que éste resulta esperanzador puesto que existen bases fundadas que hacen considerar que la función tutorial jugará un papel fundamental en el desarrollo de la actividad educativa.

En relación con el horario de dedicación a la tarea tutorial, según enseñanzas y/o etapas, Sobrado (2007) señala que se encuentran diferencias significativas. En Educación Infantil y Primaria existe una dedicación elevada a la función tutorial de 5 o más horas a la semana, siendo menor la de los niveles educativos de ESO, Bachillerato y FP.

Serrano (2009) considera que la satisfacción del tutor y tutora en su tarea es buena, aunque se encuentran decepcionados con el reconocimiento que se realiza del mismo y con su retribución económica y posibilidades de desarrollo profesional. También López (2013) señala que aunque los tutores y tutoras se consideran preparados para ejercer su labor como tal, no se sienten apoyados en ella ni su trabajo recompensado. Según Torres (2010) los profesores tutores de educación secundaria se encuentra bastante satisfecho con el desarrollo de su profesión docente aunque no se muestran satisfechos en sus relaciones con la administración educativa. La satisfacción laboral para Santos, Godás, Lorenzo y Gómez-Fraguela (2010) podría conformar decisivamente las actitudes de los docentes hacia la escuela, afectando positivamente a su motivación y desempeño

Para Castro, Expósito, López y Navarro (2015) los tutores constituyen un elemento fundamental para facilitar la colaboración, participación e implicación de las familias en la vida del centro. Reparaz y Jiménez (2015) consideran que se da una buena comunicación de la familia con el centro, siendo ésta satisfactoria para ambas partes.

\section{Marco empírico}

Con objeto de conocer las opiniones de tutores y tutoras de Educación Infantil, Educación Primaria, ESO, Bachillerato y FP de los respectivos centros educativos de las comunidades de España hemos elaborado una prueba específica que fue enviada por correo electrónico a una muestra de centros, seleccionados en función de la población de cada provincia 
y de su titularidad. Muestra que ha sido extraída del Registro Estatal de Centros Docentes no universitarios del Ministerio de Educación. Se envía una carta en la que se solicita su colaboración en el trabajo que estamos realizando respecto a la función tutorial. Se hace referencia, asimismo a la confidencialidad de la información obtenida y se ofrece poner a su disposición los resultados del trabajo una vez que estos hayan sido publicados. Se indica la autoría del estudio con nombre, dirección postal y electrónica, teléfono y centro de trabajo. Se añade el enlace para generar el cuestionario en el que se puede responder a las cuestiones planteadas, indicando que el mismo se corresponde con una aplicación de Google. Las respuestas se recogen de manera totalmente anónima en una tabla de la aplicación.

El cuestionario fue cumplimentado por 1350 tutores y tutoras, resultando ser proporcionales a la población de cada comunidad y titularidad de los centros en los que ejercen sus funciones tutoriales. El $65.0 \%$ son tutoras y el $35.0 \%$ tutores. Tienen una edad media de 46.3 años (tutoras 45.5 y tutores 47.8 , grado de significación .000, en adelante gs). Su experiencia profesional media es de 19.2 años (tutoras 18.5 y tutores 20.5, gs .001). La experiencia media como tutor o tutora es de 12.5 años en ambos casos. Según la titularidad del centro en el que ejercen su tutoría, el $81.6 \%$ lo son de públicos, el $17.4 \%$ de privados concertados y el $1.0 \%$ de privados. Dedican una media semanal de 4.9 horas a sus tareas de tutoría, empleando las tutoras 5.1 horas y los tutores 4.5 horas, (gs .000). Según la enseñanza y/o etapa, el 5.0\% son tutores y tutoras de Educación Infantil, 23.5\% de Educación Primaria, 52.1\% de ESO, 9.0\% de Bachillerato y el $10.0 \%$ de FP. Los tutores y tutoras que responden a nuestro protocolo ejercen sus funciones en centros con una media de 538 alumnos y alumnas, con un total de 727.265 alumnos y alumnas.

Según la titularidad del centro se encuentran diferencias significativas en relación con la edad de tutores y tutoras (superior en los centros públicos). La experiencia docente y la experiencia como tutor o tutora, así como las horas semanales dedicadas a la función tutorial es superior en los privados-concertados. Según el género las tutoras dedican más horas semanales a sus funciones que los tutores. 
Tabla 1

Características personales y profesionales en función del género y la titularidad del centro

1. Edad según titularidad del centro. Media. Años

\begin{tabular}{lccc}
\hline & Total & Público & Privado concertado \\
1. Total & 46.3 & 46.7 & 44.7
\end{tabular}

Gs

.005

2. Experiencia docente según titularidad del centro. Media. Años Total Público Privado concertado
1. Total
19.5
18.1
19.3

Gs

3. Experiencia como tutor o tutora según titularidad. Media. Años

$\begin{array}{rccr} & \text { Total } & \text { Público } & \text { Privado con } \\ \text { 1. Total } & 12.5 & 12.3 & 13.3\end{array}$

Gs

4. Número de horas semanales que se dedican a la función tutorial según titularidad del centro. Media.

$\begin{array}{lccc} & \text { Total } & \text { Público } & \text { Privado concertado } \\ \text { 1. Total } & 4.9 & 4.7 & 5.6 \\ \text { Gs } & & .000 & \end{array}$

5. Número de horas semanales que se dedican a la función tutorial según género. Media.

Total Tutoras Tutores
1. Total
4.9
5.1
4.5

Gs .000

Con la prueba que hemos elaborado y puesto a disposición de tutores y tutoras consultados pretendemos realizar un estudio exploratorio que nos informe sobre la actual situación de la función tutorial en las enseñanzas y etapas indicadas. Con ese objeto hemos analizado la validez de contenido mediante "juicio de expertos", cinco expertos han analizado las primeras propuestas del protocolo (Cuervo-Martínez y EscobarPérez, 2008). Respecto a la validez del constructo podemos señalar que la medida de adecuación muestral de Kaiser-Meyer-Olkin (KMO) tiene un valor de .910 y la prueba de esfericidad de Bartlett tiene un Grado de significación de .000. En el análisis factorial el método de extracción utilizado ha sido el Análisis de Componentes Principales y el método de rotación Varimax con Kaiser. La rotación converge en 3 iteraciones. Dos factores explican el $74.6 \%$ de la varianza. 
El coeficiente de fiabilidad de Cronbach de la prueba tiene un valor de Alpha $=.95$ (en la escala de 0 a 1 ) en el total de los 12 elementos analizados. La satisfacción laboral, importancia del tutor y tutora en el funcionamiento del centro y en la enseñanza del alumnado, alcanzan un coeficiente de fiabilidad de .76. Respecto al reconocimiento y colaboración en su función por parte de las administraciones educativas, equipo directivo, equipo docente y familias; la carrera profesional, retribución económica y méritos, alcanzan un coeficiente de .93 .

La prueba consta de elementos referidos a: 1 . Variables de clasificación (género, edad, tiempo de experiencia profesional, tiempo de experiencia como tutor y tutora, titularidad del centro, enseñanzas y/o etapas, número de alumnos y alumnas del centro y número de horas semanales dedicadas a la tutoría). 2. Satisfacción laboral. 3. Importancia del tutor y tutora en el funcionamiento del centro y en la enseñanza del alumnado. 4. Reconocimiento y colaboración de tutores y tutoras en sus tareas. 5. Carrera profesional. 6. Retribución económica y méritos. 7. Apoyo que reciben los tutores y tutoras por parte del profesorado del equipo docente, familias y equipo directivo el centro.

\section{Tabla 2}

Coeficientes de fiabilidad de Cronbach

Temas

Coeficiente de Cronbach

1. Satisfacción. Importancia del tutor y tutora en el funcionamiento del centro y en la enseñanza del alumnado (3 elementos) .76

2. Reconocimiento y colaboración. Carrera profesional, retribución económica, méritos (9 elementos)

\section{Resultados}

Tutores y tutoras de las distintas etapas y enseñanzas y titularidad de centros han analizado diferentes aspectos de sus funciones, los cuales consideramos en función de las variables de clasificación que hemos propuesto.

Han valorado su satisfacción en sus funciones tutoriales, tema que ha sido investigado por distintos autores (López, 2013; Santos, Godás, 
Lorenzo y Gómez-Fraguela, 2010; Serrano, 2009; Torres, 2010) considerando dicha satisfacción como adecuada.

La importancia que consideran tienen tutor y tutora para la educación del alumnado de la tutoría y para el buen funcionamiento de los centros es otro de los aspectos analizados. Acción tutorial que señalan distintos autores como centrales en la función docente y en la formación del alumnado (Álvarez, 2017; Barber y Mourshed, 2007; Cañas, Campy y Pantoja, 2005; Ceballos, 2017; García-Nieto, 2011; López, 2013; Opertti, 2017).

Se analiza, asimismo, el reconocimiento y colaboración que tutores y tutoras manifiestan tener por parte de las administraciones educativas, profesorado, equipo directivo y familias del centro. Colaboración que es analizada en sus diversos ámbitos por diversos autores (Álvarez, 2017; Castro, Expósito, López y Navarro, 2015; Godás, Lorenzo y Gómez-Fraguela, 2010; González-Palacios y Avelino-Rubio, 2016; Mateo, 2008; López, 2013; Reparaz y Jiménez, 2015; Rodríguez y Romero, 2015; Serrano, 2009; Torres, 2010; Valenzuela y Sales, 2016). Considerándose como adecuados respecto a los que les proporcionan equipos directivo y docente y familias, mientras que se entiende mejorable los que les ofrecen las administraciones educativas.

En relación con la carrera profesional de tutor y tutora se estudian sus valoraciones respecto a si en ella debe tenerse en cuenta ser o haber desempeñado la función tutorial, si ésta debe conllevar una retribución económica y si debe ser considerada en los concursos de méritos y de traslados en la función pública (y en su caso constituir merito profesional en los centros privados). Distintos autores entienden que es necesario ampliar el horario de que disponen tutores y tutoras para sus funciones, considerándose insatisfechos con su retribución económica (Bolívar, 2007; Serrano, 2009; Sobrado, 2007).

\section{Satisfacción como tutor o tutora}

La satisfacción como tutor o tutora (valorada en una escala de 0 a 10 siendo 0 "ninguna satisfacción" y 10 "la máxima satisfacción") es de 7.2 puntos. Mayor satisfacción en la función tutorial la manifiestan las tutoras, los de mayor experiencia como tutor y tutora, los de centros privados-concertados, Educación infantil y Educación Primaria, menor número de alumnos y alumnas del centro y mayor número de horas semanales dedicadas a la tarea tutorial. No se encuentran diferencias según las restantes variables analizadas. 
Algunas valoraciones del profesorado respecto a su función tutorial

María González Álvarez

Tabla 3

Satisfacción como tutor o tutora

Escala de 0 a 10 puntos. siendo 0 "ninguna satisfacción" y el 10 "la máxima satisfacción"

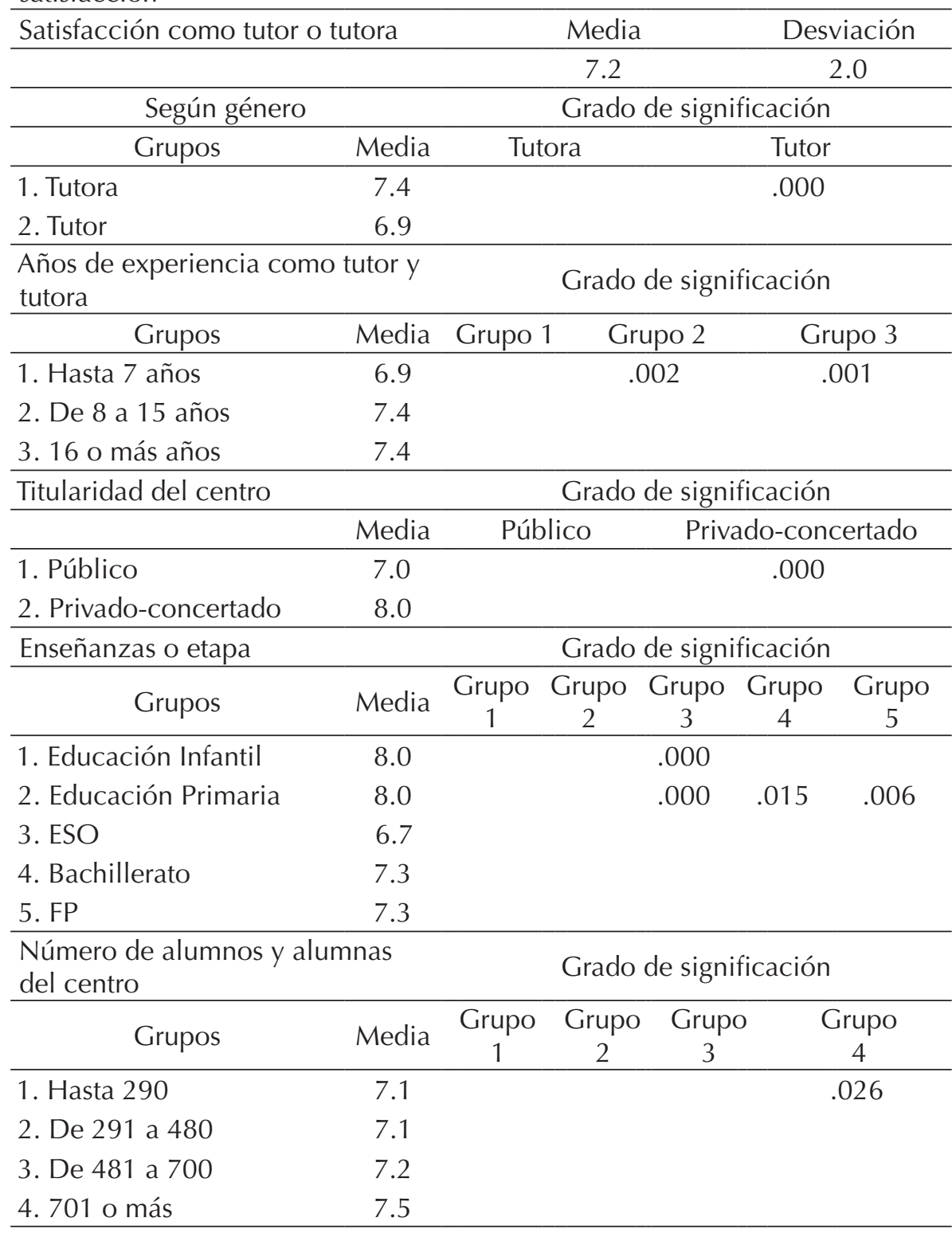




\begin{tabular}{lccc}
\hline \multicolumn{1}{c}{ Horas semanales dedicadas a la función tutorial } & \multicolumn{2}{c}{ Grado de significación } \\
\hline \multicolumn{1}{c}{ Grupos } & Media & 1 & 2 \\
\hline 1. Hasta 3 horas & 6.9 & & .000 \\
2. 4 o 5 horas & 7.1 & & .000 \\
3. 6 o más horas & 7.8 & & \\
\hline
\end{tabular}

\section{Importancia del tutor o tutora en la educación del alumnado de la tutoría}

En una escala de 0 a 10 puntos (siendo el 0 "ninguna importancia" y el 10 "mucha importancia") se valora con 8.5 la importancia que se considera tiene el tutor y tutora en la educación del alumnado de la tutoría. Es más importante para las tutoras, los de centros privados-concertados, Educación Infantil y Educación Primaria y para los que dedican seis o más horas semanales a dicha función. No se encuentran diferencias según las restantes variables.

Tabla 4

Importancia del tutor o tutora en la educación del alumnado de la tutoría

Escala de 0 a 10 puntos. siendo 0 "ninguna importancia" y el 10 "la mucha importancia"

\begin{tabular}{lcc}
\hline Importancia del tutor o tutora en a educación & Media & Desviación \\
\cline { 2 - 3 } del alumnado de la tutoría & 8.5 & 1.5 \\
\hline
\end{tabular}

\begin{tabular}{lccl}
\hline & Según género & \multicolumn{3}{c}{ Grado de significación } \\
\hline \multicolumn{1}{c}{ Grupos } & Media & Tutora & Tutor \\
\hline 1. Tutora & 8.6 & & .000 \\
2. Tutor & 8.1 & & \\
\hline Según & & & Grado designificacionn
\end{tabular}

Según titularidad del centro Grado de significación

\begin{tabular}{lcccccc}
\hline \multicolumn{1}{c}{ Grupos } & Media & Público & \multicolumn{3}{c}{ Privado-concertado } \\
\hline 1. Público & 8.4 & & & & .000 & \\
2. Privado-concertado & 8.9 & & & & \\
\hline Enseñanzas o etapa & \multicolumn{5}{c}{ Grado de significación } \\
\hline \multicolumn{1}{c}{ Grupos } & Media & Grupo & Grupo & Grupo & Grupo & Grupo \\
& & 1 & 2 & 3 & 4 & 5 \\
\hline 1. Educación Infantil & 8.9 & & & .005 & .012 & \\
2. Educación Primaria & 9.1 & & & .000 & .000 & .000 \\
3. ESO & 8.2 & & & & & \\
4. Bachillerato & 8.1 & & & & & \\
5. FP & 8.3 & & & & & \\
\hline
\end{tabular}


Horas semanales dedicadas a la función tutorial

Grado de significación

\begin{tabular}{|c|c|c|c|}
\hline Grupos & Media Grupo 1 & Grupo 2 & Grupo 3 \\
\hline 1. Hasta 3 horas & 8.2 & & .000 \\
\hline 2. 4 o 5 horas & 8.3 & & .000 \\
\hline 3. 6 o más horas & 8.9 & & \\
\hline
\end{tabular}

\section{Importancia de la función tutorial en el buen funcionamiento de los centros}

La importancia para el funcionamiento del centro de la función tutorial se valora con 8.7 puntos. Es más valorada por las tutoras, centros privados-concertados, Educación Primaria y los que dedican seis o más horas a dicha tarea. No se encuentran diferencias en relación con las restantes variables analizadas.

Tabla 5

Importancia de la función tutorial en el buen funcionamiento de los centros

Escala de 0 a 10 puntos. siendo 0 "ninguna importancia" y el 10 "la mucha importancia"

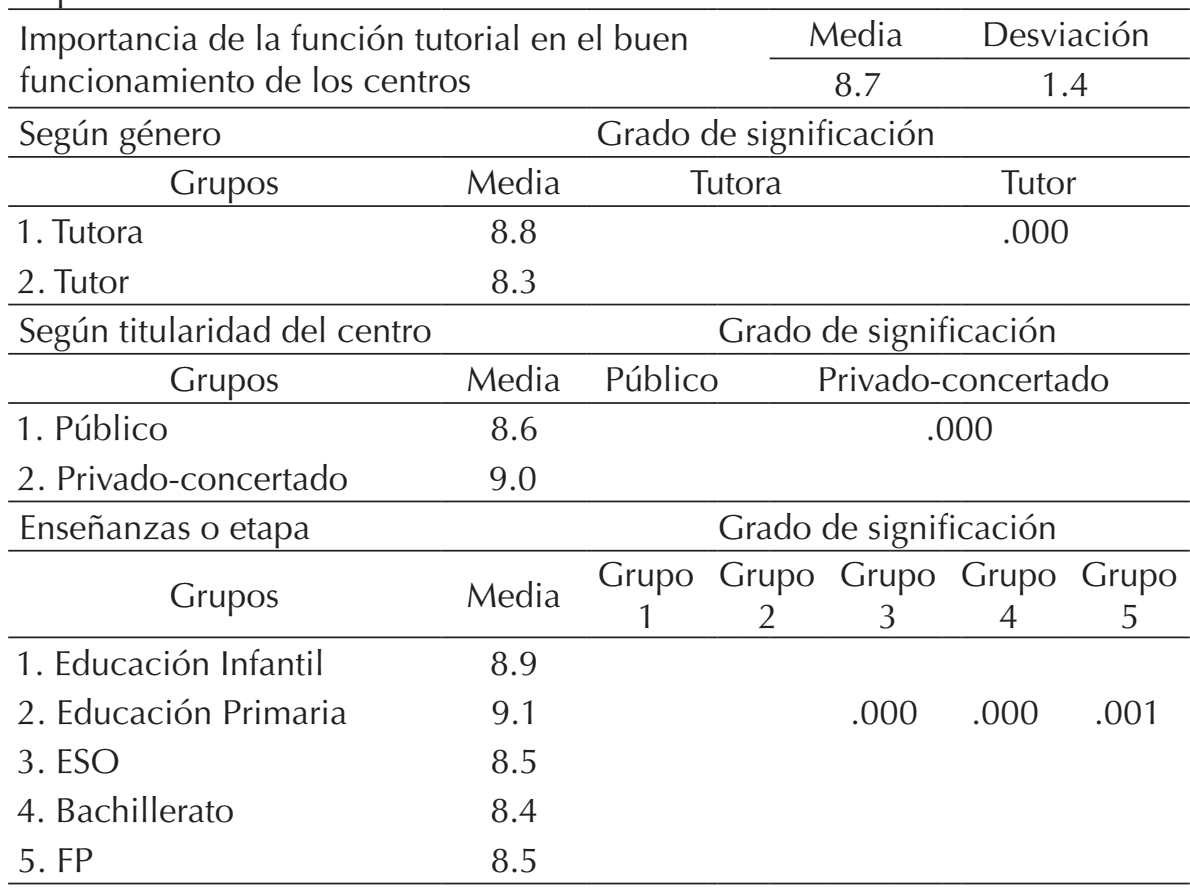




\begin{tabular}{lcccc}
\hline $\begin{array}{l}\text { Horas semanales dedicadas a la } \\
\text { función tutorial }\end{array}$ & \multicolumn{3}{c}{ Grado de significación } \\
\hline Grupos & Media & $\begin{array}{c}\text { Grupo } \\
1\end{array}$ & Grupo 2 & Grupo 3 \\
\hline 1. Hasta 3 horas & 8.4 & & & .000 \\
2. 4 o 5 horas & 8.6 & & & \\
3. 6 o más horas & 8.9 & & & \\
\hline
\end{tabular}

\section{Reconocimiento que tiene el tutor o tutora por parte de las administraciones educativas respecto a su tarea}

El reconocimiento que a juicio del profesorado que ejerce la función tutorial tiene su labor por parte de las administraciones educativas es valorado con 3.0 puntos. Es mejor valorado por los tutores y tutoras de los centros privados y privados-concertados que por los de los públicos y por los de Educación Infantil y Primaria que por los de ESO. No se establecen diferencias respecto a las restantes variables estudiadas.

Tabla 6

Reconocimiento que tiene el tutor o tutora por parte de las administraciones educativas respecto a su tarea.

Escala de 0 a 10 puntos. siendo 0 "ningún reconocimiento" y el 10 "el máximo reconocimiento"

Reconocimiento que tiene el tutor o tutora por parte de Media Desviación las administraciones educativas respecto a su tarea.

$3.0 \quad 2.8$

\begin{tabular}{|c|c|c|c|c|c|c|}
\hline \multicolumn{2}{|l|}{ Según titularidad } & \multicolumn{5}{|c|}{ Grado de significación } \\
\hline Grupos & Total & Gru & oo 1 & Grupo 2 & & цpo 3 \\
\hline 1. Públicos & 2.9 & & & .030 & & 42 \\
\hline 2. Privados-concertados & 3.4 & & & & & \\
\hline 3. Privados & 4.9 & & & & & \\
\hline Enseñanzas o etapas & & \multicolumn{5}{|c|}{ Grado de significación } \\
\hline Grupos & Media & $\begin{array}{c}\text { Grupo } \\
1 \\
\end{array}$ & $\begin{array}{c}\text { Grupo } \\
2 \\
\end{array}$ & $\begin{array}{c}\text { Grupo } \\
3 \\
\end{array}$ & $\begin{array}{c}\text { Grupo } \\
4\end{array}$ & $\begin{array}{c}\text { Grupo } \\
5\end{array}$ \\
\hline 1. Educación Infantil & 4.1 & & & .001 & & \\
\hline 2. Educación Primaria & 3.8 & & & .000 & .034 & \\
\hline 3. ESO & 2.5 & & & & & \\
\hline 4. Bachillerato & 2.8 & & & & & \\
\hline 5. FP & 3.0 & & & & & \\
\hline
\end{tabular}




\section{Reconocimiento que tiene el tutor o tutora por parte del profesorado del centro respecto a su tarea}

Respecto al reconocimiento que tienen por parte del profesorado de su trabajo lo valoan con 5.5 puntos en la escala indicada. Otorgan mayor valoración las tutoras, Educación Primaria ante Eso, Bachillerato y FP y los de centros de menos de 290 alumnos y alumnas que los de más de 480. No se encuentran diferencia respecto a las restantes variables.

Tabla 7

Reconocimiento que tiene el tutor o tutora por parte del profesorado del centro respecto a su tarea.

Escala de 0 a 10 puntos. siendo 0 "ningún reconocimiento" y el 10 "el máximo reconocimiento"

\begin{tabular}{lcc}
\hline Reconocimiento que tiene el tutor o tutora por parte & Media & Desviación \\
\cline { 2 - 3 } del profesorado del centro respecto a su tarea. & 5.5 & 3.2
\end{tabular}

\begin{tabular}{|c|c|c|c|c|c|}
\hline \multirow{2}{*}{$\begin{array}{l}\text { Según género } \\
\text { Grupos }\end{array}$} & & \multicolumn{4}{|c|}{ Grado de significación } \\
\hline & & Media & Tutora & \multicolumn{2}{|r|}{ Tutor } \\
\hline 1. Tutora & & 5.7 & & \multicolumn{2}{|r|}{.003} \\
\hline 2. Tutor & & 5.2 & & & \\
\hline \multicolumn{2}{|l|}{ Enseñanzas o etapas } & \multicolumn{4}{|c|}{ Grado de significación } \\
\hline Grupos & Media & $\begin{array}{cc}\text { Grupo } & \text { Grupo } \\
1 & 2 \\
\end{array}$ & $\begin{array}{c}\text { Grupo } \\
3 \\
\end{array}$ & $\begin{array}{c}\text { Grupo } \\
4\end{array}$ & $\begin{array}{c}\text { Grupo } \\
5 \\
\end{array}$ \\
\hline 1. Educación Infantil & 5.9 & & \multirow{5}{*}{.000} & \multirow{5}{*}{.007} & \multirow{5}{*}{.000} \\
\hline 2. Educación Primaria & 6.4 & & & & \\
\hline 3. ESO & 5.2 & & & & \\
\hline 4. Bachillerato & 5.1 & & & & \\
\hline 5. FP & 4.9 & & & & \\
\hline \multicolumn{2}{|c|}{$\begin{array}{l}\text { Número de alumnos y alumnas del } \\
\text { centro }\end{array}$} & \multicolumn{4}{|c|}{ Grado de significación } \\
\hline Grupos & Media & Grupo 1 & $\begin{array}{l}\text { irupo } \\
2 \\
\end{array}$ & Grupo 3 & $\begin{array}{c}\text { Grupo } \\
4 \\
\end{array}$ \\
\hline 1. Hasta 290 & 6.1 & & & .006 & .000 \\
\hline 2. De 291 a 480 & 5.8 & & & & .000 \\
\hline 3. De 481 a 700 & 5.2 & & & & \\
\hline 4. 701 o más & 4.7 & & & & \\
\hline
\end{tabular}


6. Reconocimiento que tiene el tutor o tutora por parte de las familias, en su caso del alumnado, de su grupo de alumnos y alumnas

La valoración media en relación con el reconocimiento que tienen tutor y tutora por parte de las familias, o en su caso del alumnado, es de 5.7 puntos. No se encuentran diferencias significativas respecto a ninguna de las variables propuestas.

\section{7. iLos docentes deben tener una carrera profesional en la que se tenga en cuenta ser o haber sido tutor o tutora?}

Tutores y tutoras valoran con 6.3 puntos el que los docentes tengan una carrera profesional en la que se tenga en cuenta ejercer o haber ejercido dicha función. Tan sólo se encuentran diferencias en función del sexo. Están más de acuerdo con dicha medida las tutoras.

\section{8. ¿El desempeño de la función tutorial debería de ser objeto de una retribución económica?}

Valoran con 6.9 puntos, tutores y tutoras, el que el desempeño de la función tutorial sea objeto de una retribución económica. Existen diferencias en función del género, las tutoras están más de acuerdo que los tutores. No se encuentran diferencias respecto a las restantes variables señaladas con anterioridad.

\section{9. ¿El ser o haber sido tutor o tutora debería ser tenido en cuenta en los concursos de méritos y de traslados en la función pública (y en su caso constituir merito profesional en los centros privados?}

Tutores y tutoras otorgan 6.9 puntos a que el ejercicio de su función se tenga en cuenta en los concursos de méritos en la función pública y constituir mérito profesional en los centros privados. Es mejor valorada la propuesta por las tutoras y por los de centros privados-concertados. No se encuentran diferencia respecto a las restantes variables. 


\section{0. ¿Cómo tutor o tutora valoran la colaboración del profesorado del equipo docente con su tarea?}

Las personas responsables de la función tutorial valoran con 6.2 puntos la colaboración que tienen por parte del profesorado con su tarea. Las tutoras valoran mejor dicha colaboración que los tutores, los de Educación Primaria que los de ESO y Bachillerato y los de centros de menor número de alumnos y alumnas (menos de 291 alumnos y alumnas) que los de más de 480 . No se encuentran diferencia respecto a las restantes variables.

Tabla 8

¿Cómo tutor o tutora cómo valora la colaboración del profesorado del equipo docente con su tarea?

Escala de 0 a 10 puntos. siendo 0 "muy mal" y el 10 "muy bien"

¿Cómo tutor o tutora cómo valora la colaboración del Media Desviación profesorado del equipo docente con su tarea?

$6.2 \quad 3.3$

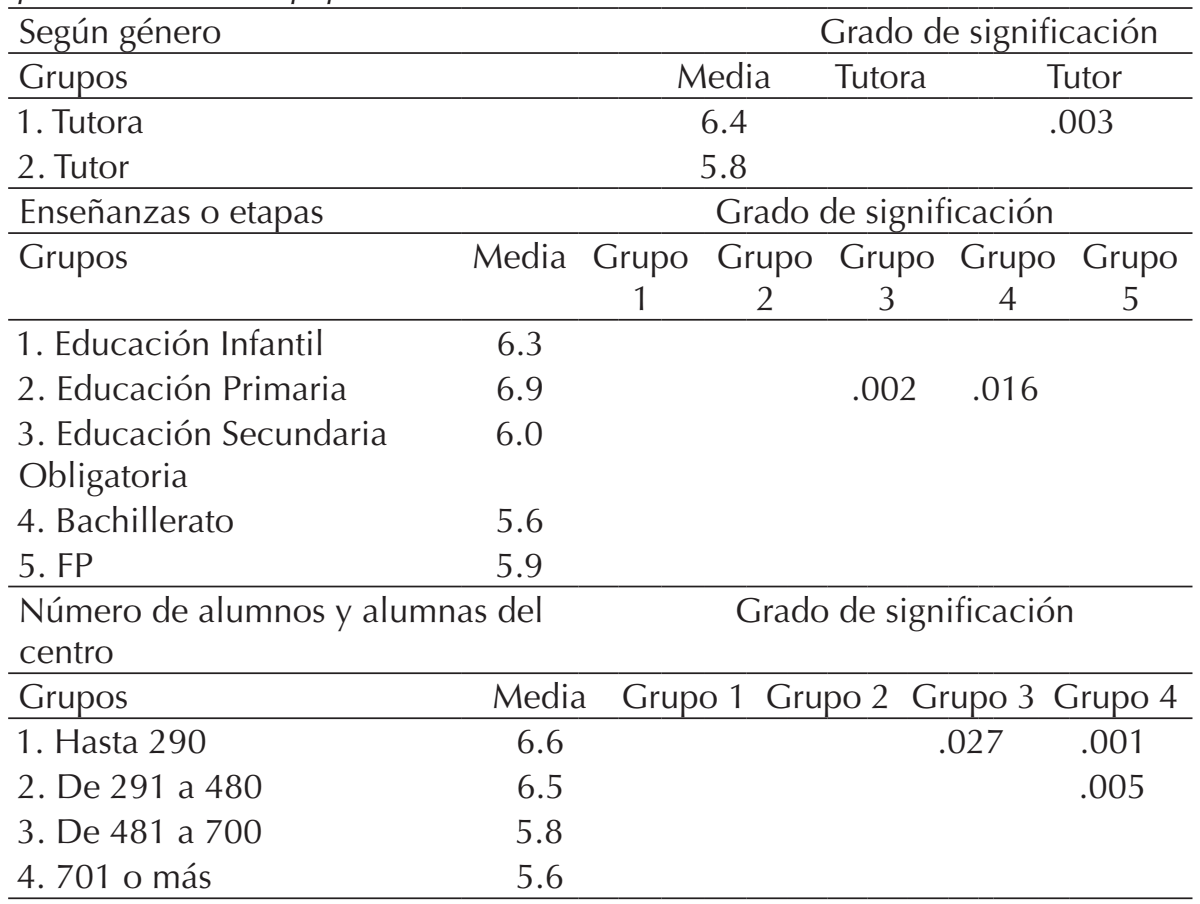




\section{1. ¿Cómo tutor o tutora cómo valora la colaboración de las familias, y en su caso alumnos y alumnas, con su tarea?}

La colaboración de las familias, y en su caso del alumnado, con las tareas del tutor y tutora es valorada por estos con 5.6 puntos. Es mejor considerada por las tutoras, Educación Primaria que ESO, Bachillerato y FP y centros de menor número de alumnos y alumnas (con menos de 290 que los de 700 o más). No se encuentran diferencias en función de las restantes variables.

Tabla 9

¿Cómo tutor o tutora cómo valora la colaboración de las familias y/o los alumnos y alumnas con su tarea?

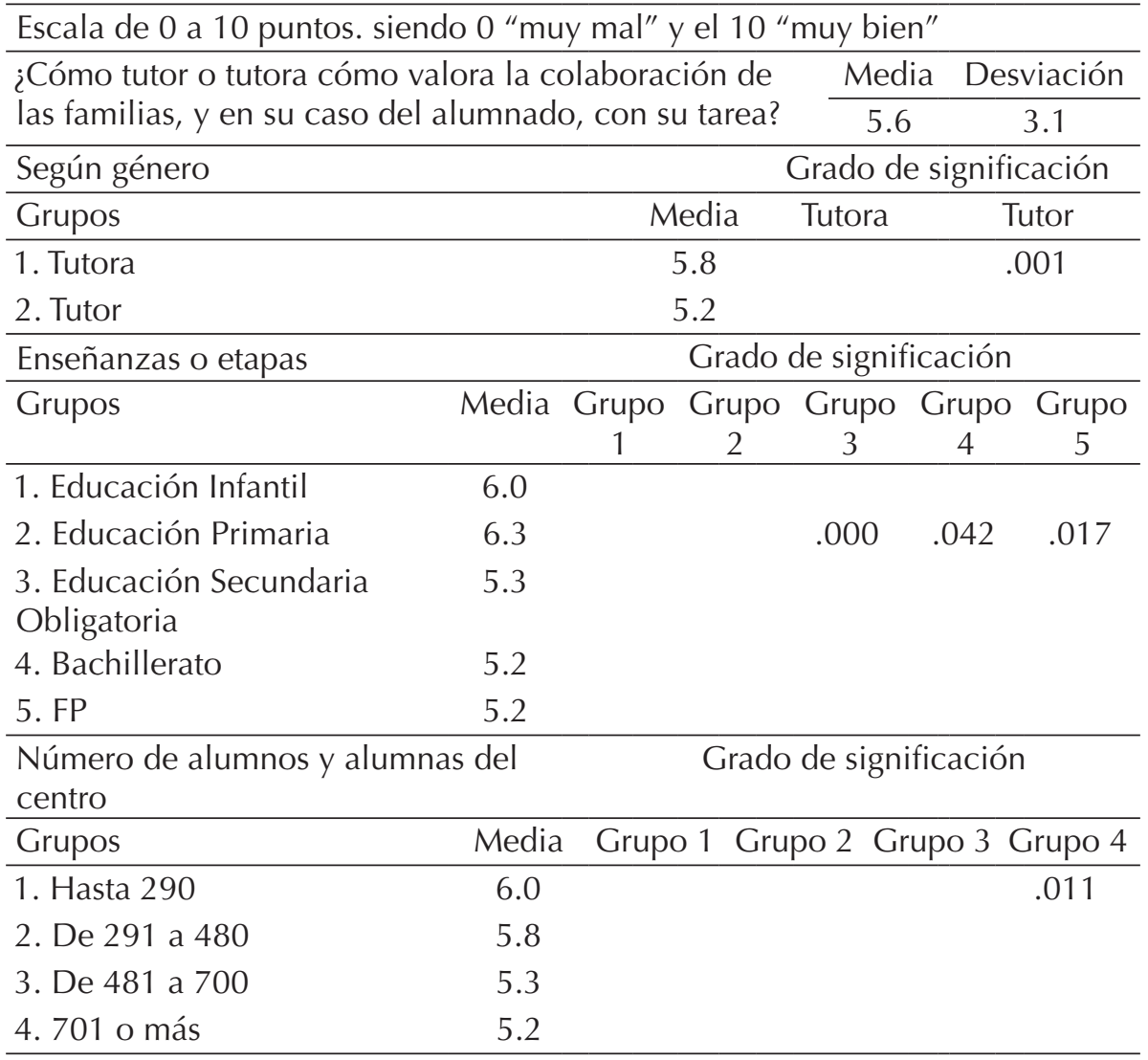




\section{2. ¿Cómo valora la colaboración que le presta el equipo directivo en su tarea de tutor o tutora?}

La colaboración que a tutores y tutoras les presta en el ejercicio de sus tareas el equipo directivo del centro la valoran con 6.2 puntos. Es mejor puntuada por las tutoras, Educación Primaria que ESO, Bachillerato y FP y los centros con menor número de alumnos y alumnas. No se encuentran diferencia respecto a las restantes variables.

Tabla 10

¿Cómo valora la colaboración que le presta el equipo directivo en su tarea de tutor o tutora?

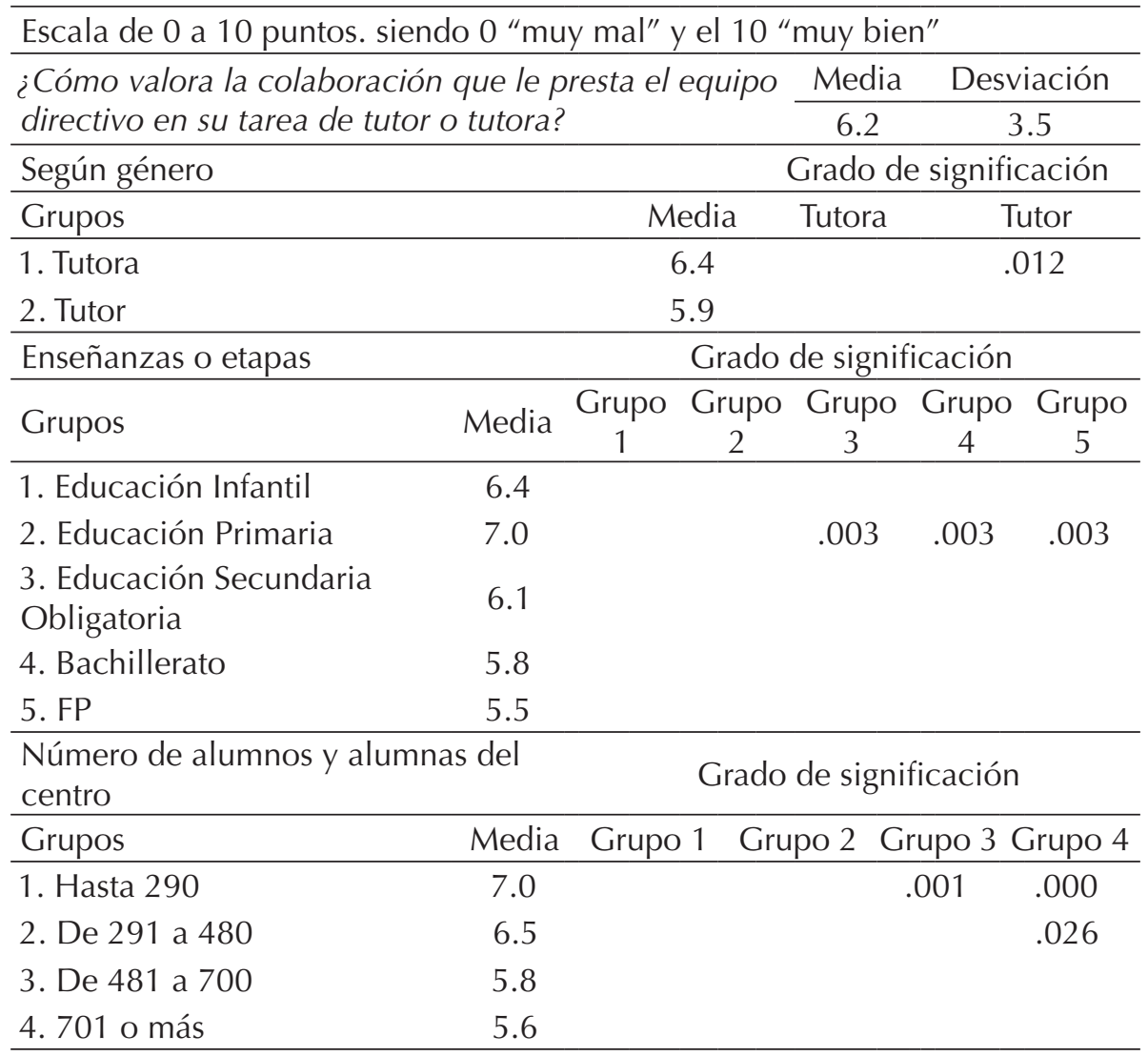




\section{Discusión y conclusiones}

La satisfacción que tutores y tutoras manifiestan tener en su función tutorial es bien valorada por ellos con 7.2 puntos (en una escala de 0 a 10, siendo 0 "ninguna satisfacción" y 10 "la máxima satisfacción"). Manifiestan tener significativamente una mayor satisfacción las tutoras, los que tienen una mayor experiencia como tutor o tutora, los que ejercen dicha función en centros privados-concertados, en Educación infantil y Educación Primaria, en centros de menor número de alumnos y alumnas y los que dedican mayor número de horas semanales a la tarea tutorial. Respecto a la satisfacción del tutor y tutora en su tarea, Serrano (2009) considera que ésta es buena. Según Torres (2010) los profesores tutores de educación secundaria se encuentran bastante satisfechos con el desarrollo de su profesión. Para Santos, Godás, Lorenzo y Gómez-Fraguela (2010) la satisfacción laboral de los docentes podría conformar decisivamente sus actitudes hacia la escuela, afectando positivamente a su motivación y desempeño.

Valoran con 8.5 puntos, en la escala señalada, la importancia del tutor y tutora para la educación del alumnado de la tutoría. Es significativamente más importante para las tutoras, los de centros privadosconcertados, Educación Infantil y Primaria y para los que dedican seis o más horas semanales a dicha función. Coincidiendo con lo señalado por Barber y Mourshed (2007) para quien la calidad de los docentes es el principal impulsor de las variaciones en el aprendizaje escolar. Docencia que, por otra parte, al estar comprometida con el proceso de aprendizaje del alumnado, debe tener en cuenta a la tutoría como parte importante de su acción (García-Nieto, 2011). Se observa, asimismo, en nuestro trabajo, la coincidencia con lo señalado por diversos autores respecto a que la tutoría es uno de los elementos integrantes de la práctica docente y factor de calidad de la enseñanza (Álvarez, 2017; López, 2013; Cañas, Campy y Pantoja, 2005).

Se valora, asimismo, con 8.7 puntos la importancia de tutor y tutora para el funcionamiento del centro. Siendo también más valorada por las tutoras, los que ejercen la tutoría en centros privados-concertados, en Educación Primaria y los que dedican seis o más horas a dicha tarea tutorial.

El reconocimiento que a juicio de los responsables de la función tutorial tiene su labor por parte de las administraciones educativas es valo- 
rado con 3.0 puntos en una escala de 0 a 10. Dicho reconocimiento es mejor valorado por los tutores y tutoras de los centros privados y privados-concertados que los de los públicos y por los de Educación Infantil y Educación Primaria que los de ESO. En consonancia con lo señalado por López (2013) que aunque los que ejercen la función tutorial se consideran preparados para ejercer su labor como tal no se sienten apoyados en ella ni su trabajo recompensado. El reconocimiento que tienen por parte del profesorado lo valoran con 5.5 puntos y el de las familias 5.7.

El que los docentes tengan una carrera profesional en la que se tenga en cuenta ejercer o haber ejercido la función tutorial se valora 6.3 puntos, en la escala señalada de 0 a 10 puntos y con 6.9 el que el desempeño de la función tutorial sea objeto de una retribución económica. La misma puntuación de 6.9 puntos es con la que se valora que ejercer la función tutorial deba ser tenido en cuenta en los concursos de méritos en la función pública y constituir mérito profesional en los centros privados. Datos que confirman lo indicado por Serrano (2009) respecto a que tutores y tutoras se encuentran decepcionados con su retribución económica y sus posibilidades de desarrollo profesional y con lo señalado por Bolívar (2007) en relación a que debe propiciarse una auténtica profesionalización del docente que debe conducir a la de un profesional del aprendizaje al tiempo que un educador.

Los responsables de la función tutorial valoran con 6.2 puntos la colaboración que reciben por parte del profesorado del equipo docente en su tarea, con 5.6 la proporcionada por las familias y con 6.2 la del equipo directivo. Podemos entender que dichas valoraciones son semejantes a lo señalado por Valenzuela y Sales (2016) respecto a que tutores y tutoras coordinan el trabajo con el equipo de profesores y profesoras y con Mateo (2008) que la tutoría es responsabilidad no sólo del profesor-tutor, sino de todos los profesores que tienen responsabilidad de un determinado grupo de alumnos. Para Castro, Expósito, López y Navarro (2015) los tutores constituyen un elemento fundamental para facilitar la colaboración, participación e implicación de las familias en la vida del centro. Reparaz y Jiménez (2015) consideran que se da una buena comunicación de la familia con el centro, siendo ésta satisfactoria para ambas partes, centro y familia.

En general, observamos que en la investigación educativa la función tutorial se considera como uno de los elementos integrantes de la práctica docente y factor de calidad de la enseñanza (Álvarez, 2017; López, 
2013; Cañas, Campy y Pantoja, 2005). Tutores y tutoras se encuentran satisfechos en el ejercicio de sus funciones a la vez que valoran positivamente el reconocimiento y apoyo que reciben de la comunidad escolar, con excepción del que les proporcionan las administraciones educativas. Entendemos que las administraciones educativas deberían prestar más apoyo a tutores y tutoras y mejorar su situación profesional. Conviene, asimismo, revisar sistemáticamente la función tutorial para adaptarla a la referida cambiante realidad socioeducativa que nos señala el profesor Álvarez (2017).

\section{Referencias}

Álvarez, M. (2017). Hacia un modelo integrador de la tutoría en los diferentes niveles educativos. Revista Educatio Siglo XXI, 35(2), 21-42. Recuperado en http://dx.doi. org/10.6018/j/298501)

Álvarez-Justel1, J. (2017). La tutoría en secundaria. Revista Educatio Siglo XXI, 35(2), 6590

Barber, M. y Mourshed, M. (2007). How the world's best performing school systems come out on top. Edit: McKinsey y Company Report.

Bereziartua, J., Intxausti, N. y Odriozola, A. (2017). Acción tutorial en la transición del alumnado de las escuelas del medio rural a la educación secundaria. Tendencias pedagógicas, 29, 189-210

Bolívar, A. (2007). La formación inicial del profesorado de secundaria y su identidad profesional. Estudios Sobre Educación, 12, 13- 30.

Cañas, A., Campoy, T.J. y Pantoja, A. (2005). La función tutorial: valoración y necesidades del profesorado. Bordón: Revista de Pedagogía, 57(3), 297-214

Castro, M., Expósito, E., Lizasoain, L., López, E., y Navarro, E. (2015). Acciones y actitudes diferenciales de los tutores y su relación con la participación de las familias. Participación Educativa, 4 (7), 29-37.

Ceballos, N. (2017). La tutoría como espacio de democracia: una mirada desde la voz de los docentes en formación. Revista Española de Orientación y Psicopedagogía, 28(1), 86-103.

Cuervo-Martínez, A. Escobar-Pérez, J. (2008). Validez de contenido y juicio de expertos: una aproximación a su utilización. Avances en Medición, 6, 27-36.

García, A. (2006): La tutoría y la relación profesor-alumno en la formación para la inserción laboral. Revista de Educación, 341, 197-211.

García-Nieto, N. (2011). La función tutorial en el ámbito educativo. Padres y maestros, 342, 5-9. Recuperado en https://revistas.upcomillas.es/index.php/padresymaestros/ article/viewFile/310/239

González-Palacios, A. y Avelino-Rubio, I. (2016). Tutoría: Una revisión conceptual. Revista de Educación y Desarrollo, 38, 57-68. 
Algunas valoraciones del profesorado respecto a su función tutorial

María González Álvarez

Ley Orgánica de 2/2006, de 3 de mayo de Educación. Ministerio de Educación.

López, E. (2013). Aproximación a la percepción y satisfacción del profesor tutor de Secundaria Obligatoria respecto a su labor. Revista de Investigación en Educación, 11(1) 77-96. Recuperado en http://webs.uvigo.es/reined/

Mateo, L. (2008). La Orientación y la Acción Tutorial en Educación Primaria. Revista digital para profesionales de la enseñanza, 10, 1-16. Recuperado en https://www. feandalucia.ccoo.es/docu/p5sd7451.pdf.

Opertti, R. (2017). 15 Claves de Análisis para Apuntalar la Agenda Educativa 2030. OIE-UNESCO. Recuperado en http://repositorio.minedu.gob.pe/handle/MINEDU/5591

Quintanal, J. (2006). La función tutorial: presente y futuro. Educación y Futuro, 15, 9-23.

Reparaz, R. y Jiménez R. (2015). Padres, tutores y directores ante la participación de la familia en la escuela. Un análisis comparado. Participación Educativa. 4(7) 3945.Recuperado en https://sede.educacion.gob.es/publiventa/descarga.action?f_codigo_agc $=17380$

Rodríguez, S. y Romero, L. (2015). La función tutorial en Educación Infantil y Primaria: desempeño profesional del profesorado. Revista Electrónica Interuniversitaria de Formación del Profesorado, 18(2), 43-55. Recuperado en http://dx.doi.org/10.6018/ reifop.18.2.219131

Santana, L.E. y Feliciano, L.A. (2006): La construcción de la acción tutorial desde las coordenadas de la investigación colaborativa. Revista de Educación, 340, 943-971.

Santos, M.A., Godás, A., Lorenzo, M. y Gómez-Fraguela, J.A. (2010). Eficacia y satisfacción laboral de los profesores no universitarios: revisión de un instrumento de medida. Revista Española de Pedagogía, 245, 151-170. Recuperado en http://reunir. unir.net/handle/123456789/3868

Serrano, C. (2009) Acción tutorial y clima escolar en los centros educativos a juicio del profesorado de Educación Secundaria Obligatoria. Pulso, 32, 95-121.

Sobrado, L. (2007). La tutoría educativa como modelo de acción orientadora: perspectiva del profesorado tutor. XXI, Revista de Educación, 9, 43-64. Recuperado en https:// dialnet.unirioja.es/descarga/articulo/4733983.pdf

Torres, J.A. (2010). Análisis del grado de satisfacción del profesorado de educación secundaria en el desarrollo de su labor docente. Contextos Educativos: Revista de Educación, 13, 27-42.

Valenzuela, C \& Sales, A (2016). Los efectos de la participación dentro del aula ordinaria. Revista nacional e internacional de educación inclusiva. 9(2), 71-86

Valle del, A. (2004). Contextos educativos y acción tutorial la tutoría integrada en la vida ordinaria del alumno: espacios y niveles de actuación en Contextos educativos y acción tutorial, 9-34. Ministerio de Educación, Cultura y Deportes. Madrid. 